\title{
HLA profile and Reiter's syndrome
}

\author{
Jane S. Schultz1,2, Armin E. Good ${ }^{1,3}$, Charles F. Sing 3 and Janet J. Kapur 1 \\ 1 Veterans Administration Medical Center and Departments of 2 Human Genetics and \\ 3 Internal Medicine, University of Michigan Medical School, Ann Arbor, Michigan, U.S.A.
}

\begin{abstract}
The analysis of the clinical and HLA profiles of 99 patients with Reiter's syndrome is reported. Antigen HLA-B27, which has previously been firmly associated with Reiter's syndrome, predisposes patients to develop disease features which reflect articular involvement. The HLA haplotype A2, B27 was found to be at an elevated frequency in our Reiter's syndrome sample, and the latter two antigens are also associated with a general increase in disease severity. Conversely, antigen BW35 appears to be protective against certain features of the syndrome. Patients with certain antigenic profiles (namely A2 and A3 together with B27) tend to develop certain syndrome manifestations earlier in the course of the disease than those with other antigens.
\end{abstract}

Received 15 July, revised 12 November, accepted for publication 10 December 1980

Key words: Disease association; HLA; human leukocyte antigens; Reiter's syndrome.

The major diagnostic criteria of Reiter's syndrome (RS) are arthritis, ophthalmitis, urethritis, and mucocutaneous lesions. A definite diagnosis is based upon arthritis of peripheral joints plus at least two other members of the tetrad. The arthritis typically involves the feet and knees and has a non-symmetric distribution. In addition, several minor criteria such as fever, diarrhea, weight loss, pain in the sacroiliac joints or spine and heel involvement have aided in diagnosis during acute attacks (Good 1977). Features that may emerge during long-term follow-up are: radiographic signs of sacroiliitis with or without chronic back pain, classical ankylosing spondylitis, heel spurs, aortitis, chronic psoriasis vulgaris, and chronic involvement or residual deformity of peripheral joints. Neuro- logical involvement (Good 1974) and iritis are features during either the acute or chronic phase of the disease.

The HLA antigen $B 27$ has a frequency of $65 \%-100 \%$ (Svejgaard \& Ryder 1977) in various series of patients with RS. This frequency may be compared to a frequency of approximately $10 \%$ in a normal population. Although it has been reported that B27 negative patients with reactive arthritis usually have a milder form of disease (Aho et al. 1974), no clear phenotypic heterogeneity between B27 negative RS patients and B27 positive patients has been definitively established.

This communication reports the results of the analysis of the clinical and HLA antigenic profiles of 99 patients with RS. We have sought to determine whether cer- 
tain clinical features of RS are predicted by any HLA-A,-B or combination of $A$ and $B$ antigens, and whether any of these features tend to cluster together in a particular group of individuals.

\section{Materials and Methods}

Ninety-nine unrelated male patients, 86 caucasians and 13 blacks, with clinical diagnoses of RS were encountered at the Ann Arbor Veterans Administration or University of Michigan Medical Centers from 1 July 1974 to 31 December 1977 . X-rays of the sacroiliac joints were available in 89 and heel X-rays in 63 .

Sixty-one definite cases of RS presented with peripheral arthritis plus two or more of the other major criteria (ophthalmitis, urethritis, and mucocutaneous lesions). In addition, 36 patients with typical peripheral arthritis and non-specific urethritis only were classified as strongly probable RS and added to the study group. Two individuals without a definite history of peripheral arthritis were included. One individual had heel pain, conjunctivitis, back pain and sacroiliitis, while the second patient presented with urethritis, sternoclavicular arthritis, balanitis and oral mucosal lesions. Ascertainment for RS was, therefore, substantially complete for the study period. Eighteen patients were encountered only during an acute attack; 81 patients were followed 18 months or more after disease onset. Patient records were screened for features of acute attacks or follow-up features listed in Table 2. The approximate years of initial occurrence of each of the acute and late features of the disease were determined from available records, written questionnaires, and/or patient interviews.

Blood specimens for tissue typing were obtained from 99 probands. One hundred forty-nine siblings, parents and/or children of 43 of these probands also contributed blood specimens to aid in the determination of proband haplotypes. Leukocytes for HLA typing are separated from whole blood samples by a modification of the method of Boyum (1968). HLA typing is performed by a minor modification of the standard NIH cytotoxicity technique (Amos 1976).

The associations between antigens, between symptoms and between antigens and symptoms were explored using the Cochran/ Mantel-Haenzel test (Landis 1979). This method was used to compute the average partial associations within tables stratified according to the length of exposure of the patient to clinical investigation. The associations within three levels of exposure (years); 1-13, 13-26, and 27 and greater, were considered. A test of heterogeneity of association among levels was taken as evidence for a bias of length of exposure on the association. Only those associations which were not significantly heterogeneous across exposure classes are reported in Tables 3-5.

\section{Results}

Table 1 contains the observed and expected number of 7 first and 13 second locus antigens in the sample studied. We have HLA typed a sample of 202 normal volunteers for 20 antigens of the $\mathrm{A}$ and $\mathrm{B}$ locus. The percentage of blacks included in our control sample corresponds with the percentage of blacks in the RS sample.

Those antigens which occur at a relative frequency $\geq 0.13$ were selected for studies of association with the clinical features of the syndrome. Those antigenic combinations which occur 11 or more times in our sample, plus the A9-B27 combination which contains two antigens with relatively high frequencies, were selected for the studies of clinical associations. The frequencies of combinations of these antigens in the patient sample did not differ from expected frequencies for a normal population. 
Table 1

Frequency of HLA antigens in Reiter's syndrome sample as compared with frequency in a normal population

\begin{tabular}{|c|c|c|c|c|c|c|c|}
\hline 1 & 23 & 30 & 1.633 & 5 & 3 & 8 & 3.125 \\
\hline 2 & 54 & 50 & 0.320 & 7 & 11 & 23 & 6.260 \\
\hline 3 & 28 & 21 & 4.666 & 8 & 14 & 17 & 0.529 \\
\hline 10 & 9 & 13 & 1.230 & 13 & 1 & 5 & - \\
\hline 11 & 6 & 11 & 2.272 & 14 & 7 & 9 & 0.444 \\
\hline \multirow[t]{5}{*}{28} & 5 & 7 & 0.571 & 15 & 3 & 12 & 6.750 \\
\hline & & & & 16 & 2 & 6 & 2.666 \\
\hline & & & & 17 & 8 & 13 & 1.923 \\
\hline & & & & 18 & 2 & 6 & 2.666 \\
\hline & & & & 40 & 7 & 7 & 0.000 \\
\hline \multicolumn{4}{|c|}{$X^{2}{ }_{\text {bat }}=12.08$} & \multicolumn{4}{|c|}{$\mathrm{X}^{2} \mathrm{11 \textrm {de }}=612.324^{* *}$} \\
\hline
\end{tabular}

* Statistically significant at the 0.01 level of probability.

Table 2

Frequency and time of appearance of RS features

\begin{tabular}{|c|c|c|c|c|c|c|}
\hline & \multicolumn{3}{|c|}{ Frequency of Response } & \multicolumn{3}{|c|}{$\begin{array}{c}\text { Appearance of RS manifestations in } \\
\text { cases relative to RS onset }\end{array}$} \\
\hline & Yes & No & Maybe & & After & $x^{2}$ \\
\hline \multicolumn{7}{|l|}{$\begin{array}{l}\text { A. Oral mucous membrane and } \\
\text { cutaneous involvement }\end{array}$} \\
\hline (1) Mouth lesions & 22 & 75 & 2 & 13 & 8 & 1.2 \\
\hline (2) Balanitis & 38 & 60 & 1 & 30 & 8 & 12.7 \\
\hline $\begin{array}{l}\text { (3) Skin or nail involvement } \\
\text { (4) Chronic skin involvement }\end{array}$ & 27 & 71 & 1 & 17 & 9 & 2.5 \\
\hline $\begin{array}{l}\text { or psoriasis } \\
\text { Average } \% \quad(24.25)\end{array}$ & 10 & 88 & 1 & 4 & 5 & - \\
\hline \multicolumn{7}{|l|}{ B. Other non-articular manifostations } \\
\hline (1) Fulminant onset, fever or weight loss & 62 & 32 & 5 & 52 & 10 & 28.4 \\
\hline (2) Diarrhea & 24 & 75 & 0 & 20 & 3 & 12.6 \\
\hline (3) Urethritis & 88 & 11 & 0 & 74 & 13 & 42.8 \\
\hline (4) Conjunctivitis & 47 & 52 & 0 & 37 & 9 & 17.0 \\
\hline (5) Aortitis & 8 & 88 & 2 & 1 & 7 & - \\
\hline $\begin{array}{l}\text { (6) Iritis } \\
\text { Average } \% \text { (41.83) }\end{array}$ & 22 & 76 & 0 & 8 & 14 & 1.6 \\
\hline \multicolumn{7}{|l|}{ C. Articular manifestations } \\
\hline (1) Heel pain & 46 & 50 & 3 & 32 & 13 & 8.0 \\
\hline (2) Peripheral arthritis & 97 & 2 & 0 & 97 & 0 & - \\
\hline (3) Acute back pain & 91 & 7 & 1 & 68 & 21 & 24.8 \\
\hline (4) Chronic peripheral joint involvement & 30 & 57 & 11 & 7 & 23 & 8.5 \\
\hline (5) Chronic back pain & 51 & 41 & 6 & 18 & 33 & 4.4 \\
\hline $\begin{array}{l}\text { (6) Ankylosing spondylitis } \\
\text { Average } \%(55.5)\end{array}$ & 18 & 80 & 1 & 1 & 16 & 13.2 \\
\hline
\end{tabular}

Average $\%$ of all manifestations - 41 
Family studies provide 72 true haplotypes for $36 \mathrm{RS}$ patients. Of the 37 haplotypes represented, only one, A2-B27 appears more than four times. This haplotype appears 18 times rather than the seven times expected based on the antigenic frequencies of $\mathbf{A} 2$ and $\mathbf{B} 27$ in this sample. This deviation from expectation is significant at the 0.05 level of probability.

Table 2 gives the frequency of each of the clinical features studied. The features have been grouped as follows: Group A - balanitis and oral mucous membrane and cutaneous and nail involvement. Allowing for regional differences due to the presence of saliva or mucous, the mucocutaneous lesions share the histopathology of pustular psoriasis, namely acanthosis, elongated rete pegs, microabscesses and hyperkeratosis (Kulka 1962), Group B - non-articular manifestations which do not share a common pathology and Group C - articular involvement. All of the features included in this group have in common either acute or chronic joint or heel pain. The frequencies of the disease manifestations listed in Table 2 serve to describe the clinical properties of the syndrome. The distribution of the reported appearance of a disease manifestation with respect to disease onset is also presented. The onset of the disease was defined as the time of appearance of peripheral arthritis or heel pain. For those cases where a test of a uniform distribution is reasonable, iritis, skin or nail involvement and mouth lesions fail to be significantly heterogeneous with respect to the onset of disease.

It is relevant for predictive purposes to know whether any of the seven selected HLA antigens or five antigenic combinations predispose an individual to develop his disease manifestation early or late in life. Taking onset of peripheral arthritis as the time of onset for RS, patients developing the syndrome before and after 30 , the median sample age, had similar frequencies of all antigens and antigenic combinations except for antigen $A 9$, which was associated with early onset $(P=0.05)$.

The associations of the clinical features within clusters and between clusters after applying the Cochran/Mantel-Haenzel correction for heterogeneity of length of exposure are found in Table 3. Table 4 shows the associations between the selected HLA

\section{Table 3}

A summary of the statistically significant positive associations between syndrome features

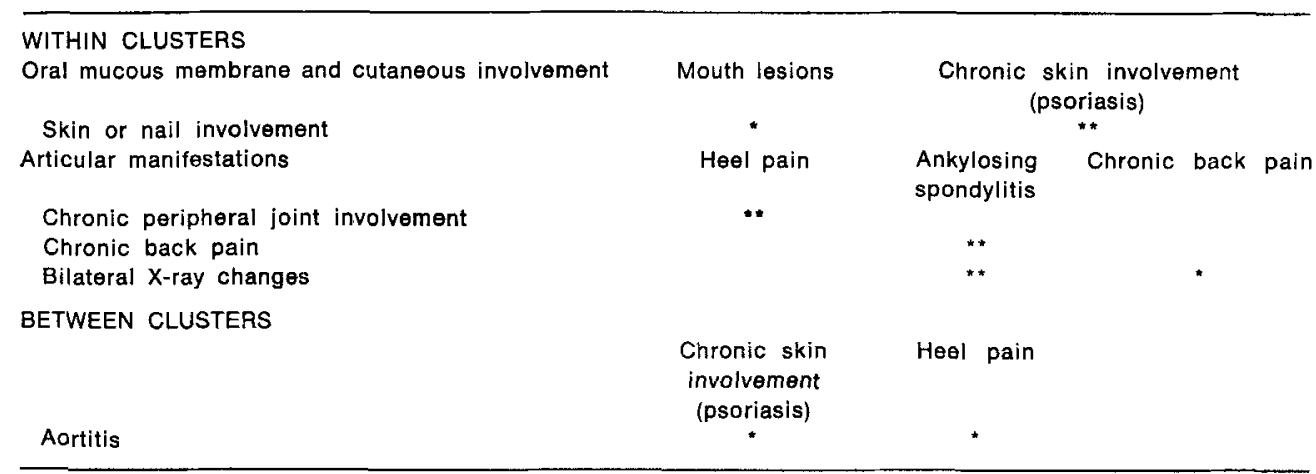

* = Probability of observing a larger maximum likelihood test criterion by chance $=\alpha=0.05$

** = Probability of observing a larger maximum likelihood test criterion by chance $=\alpha=0.01$ 
Table 4

Association of occurrence of features and appearance of features before, during or after onset of disease with selected $A$ and $B$ locus antigens

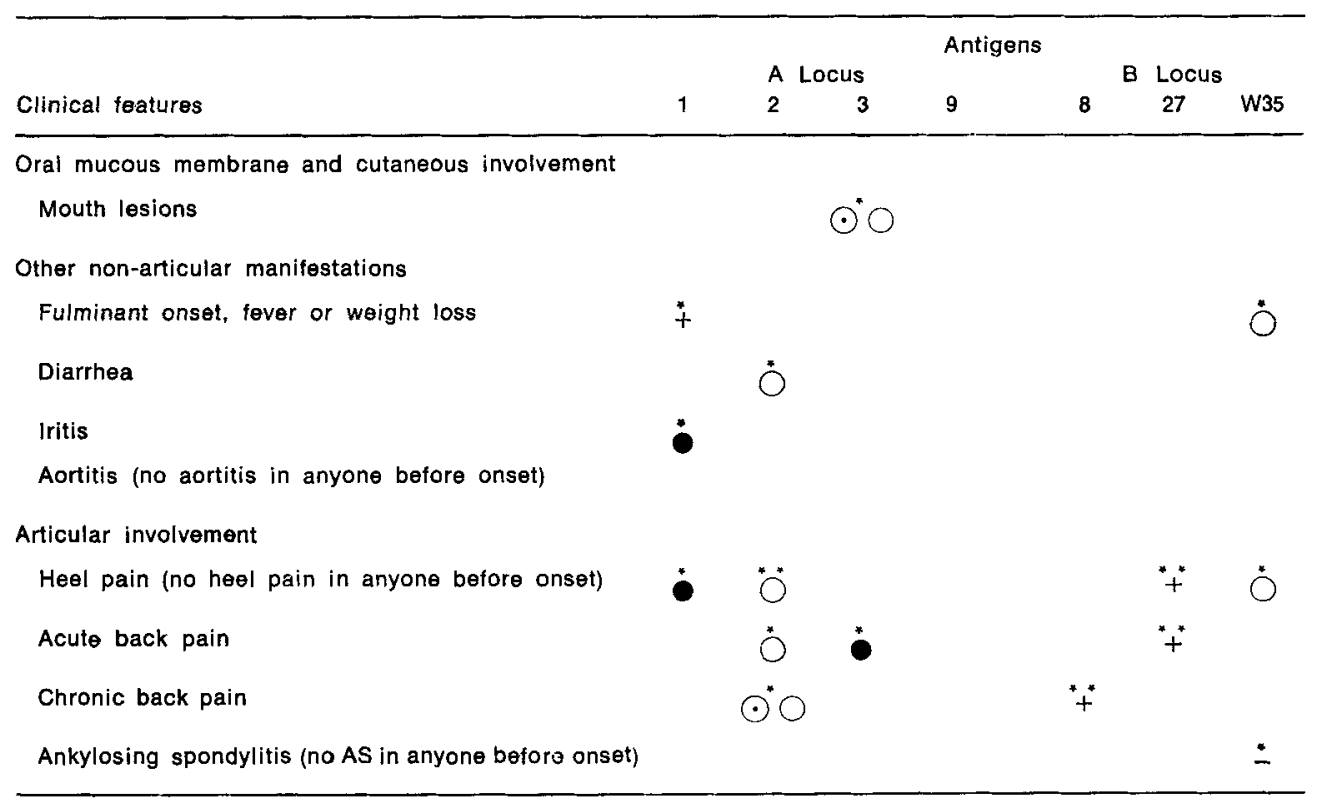

* Probability of observing a large maximum likelihood test criterion by chance $=\alpha=0.05$

* Probability of observing a large maximum likelihood test criterion by chance $=\alpha=0.01$

After - During $\bigcirc$; Before $\odot$. Positive association; - Negative association.

antigens and clinical features after the Mantel-Haenzel correction has been applied. Individuals with antigen $\mathrm{B} 27$ are more likely to develop articular features in addition to their acute peripheral joint involvement. Antigen A1 and fulminant onset with fever or weight loss, and antigen B8 and chronic back pain are also associated. A negative association of ankylosing spondylitis (AS) and antigen BW35 is shown. The results of an analysis of the relationship of age at onset of various clinical manifestations and HLA antigenic types, as shown in the same table, indicate that patients with antigens $\mathrm{A} 1,2$ or 3 or BW35 are heterogeneous in the time of occurrence of certain manifestations.

Table 5 gives the same type of analysis of associations for pairs of antigens and clinical features as is found in Table 4 for single antigens. The associations which remain consistent over the whole observation time range are those between the antigenic combination A2, B27 and heel pain, acute back pain and chronic peripheral joint involvement; antigens A1 and B27 and skin or nail involvement and chronic back pain and antigens $\mathrm{A} 1$ and $\mathrm{B} 8$ with chronic back pain. The antigen pairs $\mathrm{A} 1, \mathrm{~B} 27$ and A2, B27 are also associated with heterogeneity in time of appearance of disease features. It was established that only antigens A1 and B27 increase the risk of developing a larger total number of clinical features (11-14) of RS when found separately or in combination. No other antigens or 
Table 5

Association of occurrence of features and appearance of features beforc, during and after onset of disease with selected $A$ and $B$ locus antigenic pairs

\begin{tabular}{|c|c|c|c|c|c|}
\hline Clinical feature & 1,27 & 2,27 & 3,27 & 2,35 & 1,8 \\
\hline \multicolumn{6}{|c|}{ Oral mucous membrane and cutaneous involvement } \\
\hline Skin or nail involvement & $\ddot{+}$ & & & & \\
\hline \multicolumn{6}{|l|}{ Other non-articular manifestations } \\
\hline \multicolumn{6}{|l|}{ Fulminant onset, fever or weight loss } \\
\hline \multicolumn{6}{|l|}{ Iritis } \\
\hline \multicolumn{6}{|l|}{ Articular involvement } \\
\hline \multicolumn{6}{|l|}{ Heel pain } \\
\hline Acute back pain & & + & & & \\
\hline Chronic peripheral joint involvement & & + & & & \\
\hline Chronic back pain & $\stackrel{*}{+}$ & & & & + \\
\hline
\end{tabular}

* Probability of observing a large maximum likelihood test criterion by chance $=\alpha=0.05$

* Probability of observing a large maximum likelihood test criterion by chance $=a=0.01$

After - ; During $O$; Before $\odot$. Positive association; - Negative association.

antigenic combinations are predictive of the number of clinical features which develop in these patients.

\section{Discussion}

Our data have enabled us to draw certain conclusions about the inter-relationships between the clinical features of RS and their heterogeneity with respect to certain HLA-A and -B locus antigens and combinations of these antigens. As one would anticipate from consideration of the literature, the deviation of the observed frequency of $\mathrm{B} 27$ antigen from its expectation in normals $\left(\mathrm{X}^{2}{ }_{1 \mathrm{df}}=578.00\right)$ is the largest contributor to the overall poorness of fit $\left(\mathrm{X}^{2}{ }_{11 \mathrm{df}}=\right.$ 612.324) of the data to the frequencies expected in a random sample of healthy individuals. The excess of B27 is compensated for by deficiencies of all other B locus antigens tested. The elevated frequency of the A2, B27 haplotype in RS is in agree- ment with the data of Terasaki \& Mickey (1975). Even though the A2, B27 haplotype exceeds the expected frequency, the simple presence of these two antigens in a single individual does not appear at an elevated frequency.

Our arrangement of clinical features into clusters was made to be consistent with clinical diagnostic procedure. The groupings of clinical features were those which a physician might generate when presenting a clinical diagnosis. A dichotomy in frequency of occurrence among these clustered characteristics is apparent. Articular manifestations occur on the average in more than half of RS patients $(56 \%)$, while single features of oral mucous membrane involvement are present in less than $25 \%$ of all patients. Clearly there are two separate groups of disease symptoms in this sample, those which are most common and those which are less common. More than $40 \%$ of the test population also show manifestations 
which are outside of these two groups (classed as other non-articular manifestations).

We found considerable heterogeneity in time of reporting of disease features in relationship to disease onset. Mucous membrane and other non-articular manifestations tended to occur more often concurrently with the disease onset, while the features of articular involvement varied with respect to time of onset. Heel pain and acute back pain appeared more often at onset, while chronic peripheral joint involvement, chronic back pain and AS tended to appear after the onset of the disease. In this study, most features occurred acutely either at or after disease onset.

The large amount of clinical data available from this study enables us to ask which disease features tend to aggregate in individuals. Some features are yet to appear in those individuals who have been observed for short periods, since correlations between features made before the Mantel-Haenzel correction was applied differed markedly from the correlations outlined in Table 3 . The highest degree of association was shown to occur within the articular manifestations cluster. Chronic peripheral joint involvement was associated only with heel pain, and, as expected, chronic back pain and bilateral sacroiliac $\mathrm{X}$-ray changes were associated with AS. It appears, therefore, that features of articular involvement may be separated into two groups; those involving the spine and those involving the periphery. The association between skin or nail involvement and chronic skin involvement was the strongest $(P<0.0003)$ of all associations and certainly unlikely to be a chance association. Skin or nail involvement and mouth lesions, which are significantly associated, also have a common pathophysiology. Unexpected associations across clusters were between aortitis and heel pain and chronic skin involvement.

Not only is antigen B27 a predictor of disease occurrence, but it also predisposes
A

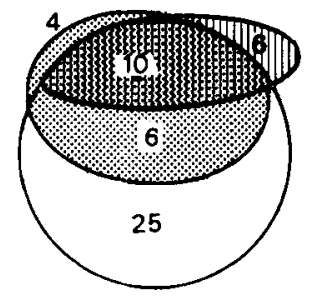

B

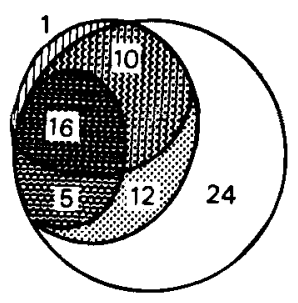

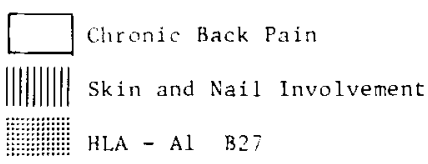

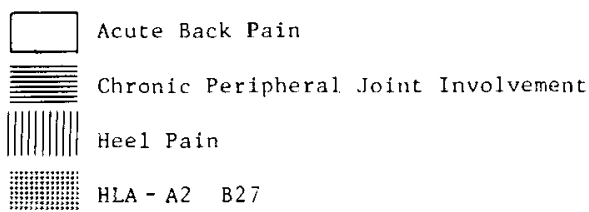

Fig. 1. Associations of syndrome manifestations and antigen pairs in Reiter's syndrome. 
an RS patient to heel pain and acute back pain (Table 4). When both antigens $A 2$ and B27 are present, there is a tendency to chronic peripheral joint involvement (Table 5 ), not evident when $B 27$ or A2 occurs independently. However, patients with A2 tend to acquire articular involvement early in the disease course.

Although antigen $\mathrm{A} 1$ is not associated with chronic back pain or skin and nail involvement, it shows an association with these characteristics when antigen B27 or B8 (in the case of chronic back pain) is present as well. Conversely, although antigen A1 is associated with fulminant onset of the disease with fever or weight loss, no particular B locus antigen is related to increased likelihood of development of these characteristics. Patients with antigen A1 appear to have relatively late development of RS.

Of particular interest is the negative association between antigen BW35 and AS. It appears that this antigen confers a protective effect against the development of AS secondary to RS, and may account for the reduced severity of $\mathrm{RS}$ in blacks (Good 1979) who have a high frequency of this antigen.

The Venn diagrams in Figure 1 give a visual summarization of the interrelationships of two cross cluster associated features and the antigen pair A1, B27, and of three within cluster associated features and antigen pair A2, B27. The cross cluster association is shown in Figure 1-A. Sixteen of 41 individuals with chronic back pain are HLA-A1, B27. Ten of these individuals also have skin or nail involvement. There are six individuals with skin or nail involvement who do not report chronic back pain. Interestingly, these individuals also lack the A1, B27 combination. Only 4 of the 20 individuals who have A1, B27 do not have chronic back pain and skin or nail involvement.
As can be seen in Figure 1-B, the intracluster relationship of acute back pain, chronic joint involvement and heel pain with the antigenic combination A2, B27 is even more pronounced. All 43 RS patients with A2, B27 have had acute back pain, 26 of the 43 have heel pain and 21 of the 43 have chronic joint involvement. There are 16 patients who share all three clinical characteristics and the antigenic combination. These relationships are all independent of length of time observed.

It has frequently been hypothesized that HLA antigenic profile might influence disease development and severity, particularly in those multifaceted diseases in which a clear association with one HLA antigen has been shown. We have demonstrated that HLA antigenic profile does not show random distribution with some clinical manifestations of RS. HLA analysis may therefore lead to the identification of disease subsets with different phenotypic expression, the consequence of genetic-environmental interactions. These data are consistent with the hypothesis that alleles at the HLA locus modify factors primarily responsible for the onset or course of RS. Ultimately the clinician may be able to design appropriate therapeutic regimens based on early manifestations and HLA type.

\section{Acknowledgments}

We wish to acknowledge the excellent technical assistance of Ms. Karen Kral, Ms. Dierdre Smith, Ms. Sheryl Elston and Mr. Kenneth Kral. Supported by the Medical Research Service of the Veterans Administration.

\section{References}

Aho, K., P. Ahvonen, A. Lassus et al. (1974). Arthr. Rheum. 17, 521-526.

Amos, D. B. (1976). Cytotoxicity testing, NIAID Manual of Tissue Typing Techniques 
(DHEW Publication No. (NIH) 76-545), ed. J. G. Ray Jr, D. B. Hare, P. D. Pedersen, and D.I. Mullally. Bethesda, Research Resources Branch (NIAID), pp. 25-28.

Boyum, A. (1968). Separation of leucocytes from blood and bone marrow. Scand. J. clin. Lab. Invest. 21, Suppl. 97.

Good, A. E. (1974). Reiter's disease: A review with special attention to cardiovascular and neurologic sequellae. Sem. Arthr. Rheum. 3, 253-286.

Good, A. E. (1977), Reiter's disease. Postgrad. Med. 61, 153-158.

Good, A. E. (1979), Reiter's syndrome: longterm follow-up in relation to development of ankylosing spondylitis. Ann. rheum. Dis. 38, Suppl. 39-45.

Kulka, J. P. (1962). The lesions of Reiter's syndrome. Arthr. Rheum. 5, 195-201.
Landis, J. R. (1979). Introduction to the Theory of Statistics. New York, MoGraw Hill, p. 314.

Svejgaard, A. \& L. P. Ryder (1977). Associations between HLA and disease. HLA and Disease, ed. I. Dausett and A. Svejgaard. Munksgaard, Copenhagen, pp. 46-71.

Terasaki, P. I. \& M. R. Mickey (1975) HLA haplotypes of 32 diseases. Transplant. Rev. 22, 105-119.

Address:

Jane S. Schultz, Ph.D.

Research Service (151)

Veterans Administration Medical Center 2215 Fuller Road

Ann Arbor, Michigan 48105

U.S.A. 